\title{
Expressions and significances of CTSL, the target of COVID-19 on GBM
}

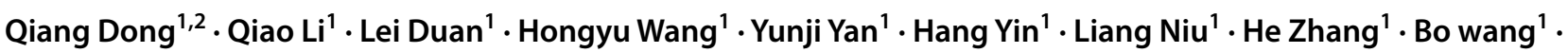

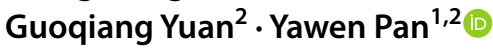

Received: 20 June 2021 / Accepted: 22 October 2021 / Published online: 22 November 2021

(C) The Author(s), under exclusive licence to Springer-Verlag GmbH Germany, part of Springer Nature 2021

\begin{abstract}
Introduction Cathepsin L (CTSL) is a kind of the SARS-entry-associated CoV-2's proteases, which plays a key role in the virus's entry into the cell and subsequent infection. We investigated the association between the expression level of CTSL and overall survival in Glioblastoma multiforme (GBM) patients, to better understand the possible route and risks of new coronavirus infection for patients with GBM.

Methods The expression level of CTSL in GBM was analyzed using TCGA and CGGA databases. The relationship between CTSL and immune infiltration levels was analyzed by means of the TIMER database. The impact of CTSL inhibitors on GBM biological activity was tested.

Results The findings revealed that GBM tissues had higher CTSL expression levels than that of normal brain tissues, which was associated with a significantly lower survival rate in GBM patients. Meanwhile, the expression level of CTSL negatively correlated with purity, B cell and $\mathrm{CD} 8^{+} \mathrm{T}$ cell in GBM. CTSL inhibitor significantly reduced growth and induced mitochondrial apoptosis.

Conclusion According to the findings, CTSL acts as an independent prognostic factor and can be considered as promising therapeutic target for GBM.
\end{abstract}

Keywords Glioblastoma $\cdot$ CTSL $\cdot$ COVID-19 $\cdot$ Prognosis $\cdot$ Immune cell infiltration

\section{Introduction}

Coronavirus disease 2019 (COVID-19) is highly infectious and transmitted mainly by inhaling droplets or aerosols released by an infected individual and possibly by a feco-oral transmission route, has accumulated 160,317,950 confirmed cases globally until mid-May 2021 (https://www.world ometers.info/coronavirus/). Understanding the mechanism of action of the virus is an important step in determining the best treatment. It has been reported that SARS-CoV2 enters

Qiang Dong and Qiao Li contributed equally to this work.

Guoqiang Yuan

yuangq08@1zu.edu.cn

$\triangle$ Yawen Pan

panyw2018@163.com

1 Department of Neurosurgery, Lanzhou University Second Hospital, Lanzhou 730030, Gansu, People's Republic of China

2 Key Laboratory of Neurology of Gansu Province, Lanzhou 730030, Gansu, People's Republic of China host cells via spike proteins that bind to the angiotensinconverting enzyme 2 (ACE2) membrane-bound protein (Gemmati et al. 2020). After binding to target cells, the $S$ protein is cleaved into two subunits: $\mathrm{S} 1$ and $\mathrm{S} 2$ by TMPRSS2 and host cell proteases such as cathepsin L (CTSL), which promotes viral entry into the cell by inducing membrane fusion and endocytosis of several coronaviruses. This cleavage of $\mathrm{S}$ protein by host proteases is critical for viral activation and subsequent infection (Hou et al. 2020; Hoffmann et al. 2020). Infection by SARS-CoV-2 results in significant morbidity and mortality. While the lung is the major organ of infection, some studies implied that SARS-CoV-2 might invade the CNS, causing neurological disorders (Narayanappa et al. 2021). Neurological symptoms, such as headache, dizziness, and impaired consciousness as well as symptoms involving the cranial nerves have been reported in COVID19 patients (Achar and Ghosh 2020).

CTSL, a member of the lysosomal cysteine protease family, plays a role in lysosomal protein degradation in most cell types (Nakagawa 1998). CTSL expressing on the surface of cancer cells and secreted into the extracellular matrix to 
degrade extracellular matrix components. CTSL preferentially cleaves the peptide bond between the aromatic residue at the $\mathrm{p} 2$ position and the hydrophobic residue at the P3 position (Brady et al. 2000). The acidic environment in lysosomes promotes the activation of proteolytic enzymes. In addition, the locally acidic environment caused by anaerobic glycolysis also activates extracellular CTSL, which encourages the invasion of cancer cells into surrounding tissues, blood and lymphatic vessels, and the metastasis of tumor tissue to distant tissues (Sudhan and Siemann 2013). Previous studies have shown that the high expression of CTSL significantly promotes the proliferation, invasion, migration, drug resistance and angiogenesis in lung cancer, gastric cancer, ovarian cancer, breast cancer, glioma and other malignant tumors (Yao et al. 2018; Sigloch et al. 2017). Shan et al. (2016) found that the over-expressed transcription factor Forkhead box O3a (FOXO3a) in gastric cancer cells can activate the promoter of CTSL, inhibit the expression of E-cadherin, and promote the development of gastric cancer. CTSL inhibitors can reduce the tumor model's ability to form blood vessels (Sudhan et al. 2016).

The pathogenesis of tumors is relatively complex, involves a variety of pathophysiological processes, and is affected by a variety of factors, among which the immune status of the body cannot be ignored. In the process of tumor occurrence and development, the immune response of the organism is often weakened (Leskowitz et al. 2015). Due to the low immune function of patients with malignant tumors and the suppression of the systemic immune system caused by anti-tumor treatments such as radiotherapy and chemotherapy or surgery, tumor patients are more susceptible to SARS-CoV2 than non-tumor patients (Addeo and Friedlaender 2020). In addition, the expression of SARSCoV2 receptors (ACE2, CTSL and TMPRSS2) significantly increased in many kinds of tumors, which makes viral entry into the cell and cancer patients more susceptible to SARSCoV2 (Bao et al. 2020).

Analyses of the expression and related biological processes of CTSL in GBM are useful to aid in the understanding of COVID-19 pathogenesis and the development of therapeutic strategies. In the present study, CTSL expression was identified using TCGA and GEPIA databases. Furthermore, the diagnostic and prognostic values of CTSL in GBM were determined. Subsequently, we evaluated whether CTSL can be regarded as a therapeutic target for GBM.

\section{Materials and methods}

\section{Reagents}

CTSL inhibitor 1 was purchased from Selleck Chemicals (Shanghai, China). Cell Counting Kit-8 (CCK-8) was purchased from Dojindo Molecular Technologies (Kumamoto, Japan). PE Annexin V apoptosis detection commercial kit were purchased from BD Biosciences (Shanghai, China). Cell-Light EdU Apollo567 in Vitro Kit was purchased from Guangzhou Ruibo Biotechnology Co. LTD (Guangzhou, China). Anti-CTSL, anti-Bax, anti-Bcl-2 and anti-MMP-9 antibodies were obtained from Proteintech (Wuhan, China). Anti-GAPDH was purchased from Abcam (Cambridge, UK).

\section{Data collection}

The Cancer Genome Atlas (TCGA; https://www.cancer.gov/ tcga) was used to obtain GBM RNA-seq data. A total of 35 GBM and 16 normal brain tissue samples were acquired with signed informed consent from Lanzhou University Second Hospital. Normal brain tissue samples were obtained from patients without glioma who underwent surgery for other reasons, including cerebral trauma. All procedures involving human samples were approved by the Ethics Committee of Lanzhou University Second Hospital.

\section{Survival analysis}

The prognostic value of CTSL was analyzed using CGGA (http://www.cgga.org.cn/). The overall survival (OS) rates of the patients in the high-level and low-level GBM groups were evaluated using Kaplan-Meier analysis.

\section{TIMER database analysis}

TIMER is a database (https://cistrome.shinyapps.io/timer/) for systematic analysis of immune information of various types of tumors (Deng et al. 2020). The TIMER database contains data from a total of 10,897 samples of 32 cancers from TCGA and can assess the abundance of tumor immune infiltration. The "Gene" module was used to analyze the correlation between the expression level of CTSL and the abundance of immune cell infiltration in GBM.

\section{Quantitative Real-Time PCR}

Total RNA was extracted from tissues using TRIzol reagent (Invitrogen). Then, reverser anscribed with the reverse transcription kit (RR037A, Takara Bio, Japan). The expression of mRNAs of CTSL were determined with a LightCycler (RR390Q, Takara Bio, Japan) using TB SYBR green Premix Ex Taq II (Takara Bio, Japan). And the results were analyzed by $2^{-\Delta \Delta C T}$ method. The following primers were used: CTSL forward, 5'- GAAAGGCTACGTGACTCCTGTG -3' and reverse, 5'- CCAGATTCTGCTCACTCAGTGAG -3'; GAPDH, forward 5'-GGACCTGACCTGCCGTCTAG -3' and reverse, 5'-TAG CCCAGGAGGATGCCCTTGAG-3'. 


\section{CCK8 and Edu assays}

U251 cells were inoculated in 96-well plates and treated with BCA for $24 \mathrm{~h}, 48 \mathrm{~h}$ and $72 \mathrm{~h}$. Each well is added 10 $\mu \mathrm{L}$ of CCK8, incubated for $2 \mathrm{~h}$ at $37{ }^{\circ} \mathrm{C}$ incubator, and then detected absorbance with a microplate reader. U251 cells were seeded in 96-well plates, each well is added $100 \mu \mathrm{L}$ of $50 \mathrm{uM}$ Edu solution, incubated for $2 \mathrm{~h}$ at $37{ }^{\circ} \mathrm{C}$ incubator, and then $4 \%$ Paraformaldehyde fixation. Wash with PBS for three times, and each well is added $100 \mu \mathrm{L} 1 \times$ Hoechst 33342 solution, incubated at $37^{\circ} \mathrm{C}$ in the dark for $30 \mathrm{~min}$, then observed and analyzed under a fluorescence microscope.

\section{Apoptosis analysis}

U251 cells were treated with 0,50 , and $100 \mu \mathrm{mol} / \mathrm{L}$ BCA for $48 \mathrm{~h}$. Then, U251 cells $\left(1 \times 10^{6}\right)$ were collected, after which $5 \mu \mathrm{L}$ of PE Annexin V and $5 \mu \mathrm{L}$ of 7-AAD were added. Gently vortex the cells and incubate at $25^{\circ} \mathrm{C}$ in the dark for $15 \mathrm{~min}$ and the suspension was analyzed by flow cytometry (BD FACSCanto ${ }^{\mathrm{TM}}$ low cytometry, USA).

\section{Wound healing assay}

CTSL inhibitor-treated U251 cells were inoculated into a six-well plate. When the cells reach a confluence of $70-80 \%$, were gently and slowly scratched with a new $200 \mu \mathrm{L}$ pipette tip. The relative distance of the cells migrating was monitored and measure using a bright-field microscope at 0,12 and $24 \mathrm{~h}$.

\section{Transwell cell migration assay}

Transwell chambers membrane was pre-coated with diluted Matrigel (1:8 BD Biosciences). About $1 \times 10^{6}$ cells in 100 $\mu \mathrm{L}$ serum-free medium were added into the top chambers, and $600 \mu \mathrm{L}$ DMEM medium was added into the lower chamber. After $24 \mathrm{~h}$. the chambers were washed and cells were fixed in $4 \%$ paraformaldehyde, and subsequently stained with $0.1 \%$ crystal violet. Cell invasion assay was performed as above except used the cell culture inserts coated with Matrigel (BD Biosciences).

\section{Western blot}

U251 cells $\left(2 \times 10^{6}\right)$ were lysed using RIPA buffer (Solarbio, China). The lysed cells were centrifuged at $12,000 \times g$ for $10 \mathrm{~min}$ at $4{ }^{\circ} \mathrm{C}$. Proteins were loaded on $10 \%$ SDS-PAGE gels and transferred onto PVDF membranes. The band was visualized by enhanced chemiluminescence with imageQuant LAS 500 system.

\section{Statistical analyses}

SPSS version 23.0 (IBM, Armonk, NY, USA), GraphPad Prism 8.0 (GraphPad, San Diego, CA, USA) and R software v4.0.3 (R Foundation for Statistical Computing, Vienna, Austria) were used to perform statistical analyses and graphing. Survival curves were estimated using the Kaplan-Meier method with the log-rank test. $P<0.05$ was considered significant.

\section{Results}

\section{Expressions of CTSL in GBM}

To elucidate the expression of CTSL in GBM, we analyzed the TCGA and GEPIA database. The level of CTSL expression was markedly upregulated in GBM $(P<0.05$; Fig. 1A, B). The expression level of CTSL was confirmed using RTqPCR and western blotting in GBM tissues $(n=35)$ and normal brain tissues $(n=16)$ to confirm the GEPIA database findings. The results showed that the level of CTSL was significantly increase in GBM $(P<0.05$; Fig. $1 \mathrm{C}, \mathrm{D})$. Meanwhile, the data was consistent with TCGA and GEPIA database analysis. The correlation between the clinicopathological characteristic and the expression of CTSL was evaluated in the cases of $35 \mathrm{GBM}$ patients. The median mRNA level of CTSL was used as a cut-off value. However, the CTSL expression levels was not significantly associated with any of the other parameters assessed, including age, sex, and Karnofsky Performance Status $(P>0.05)$ (Table 1).

\section{The effect of the expression of CTSL on prognosis in GBM}

To determine whether CTSL can been regarded as an independent prognostic factor for GBM patients, the correlation between OS and the expressions of CTSL was evaluated in the cases of GBM patients using Kaplan-Meier analysis and log-rank tests. The results indicated that patients with high expression of CTSL had a lower OS rate than patients with low expression of CTSL ( $P=0.035$; Fig. 2D). In addition, we further verified the results coming from TCGA and CGGA databases (Fig. 2B, C). To further identify the diagnostic value of CTSL in GBM patients, ROC curve analysis was performed. The results showed (Fig. 2E) that the AUC for 1, 3, and 5 years were 0.698 (95\% confidence interval [CI] 0.622-0.733), 0.627 (95\% confidence interval [CI] 0.494-0.76), and $0.337(95 \%$ confidence interval $[\mathrm{CI}]=0.259-0.416$ ), respectively (Fig. 2E). It is suggested that the CTSL has higher prognostic value in the GBM. 
A

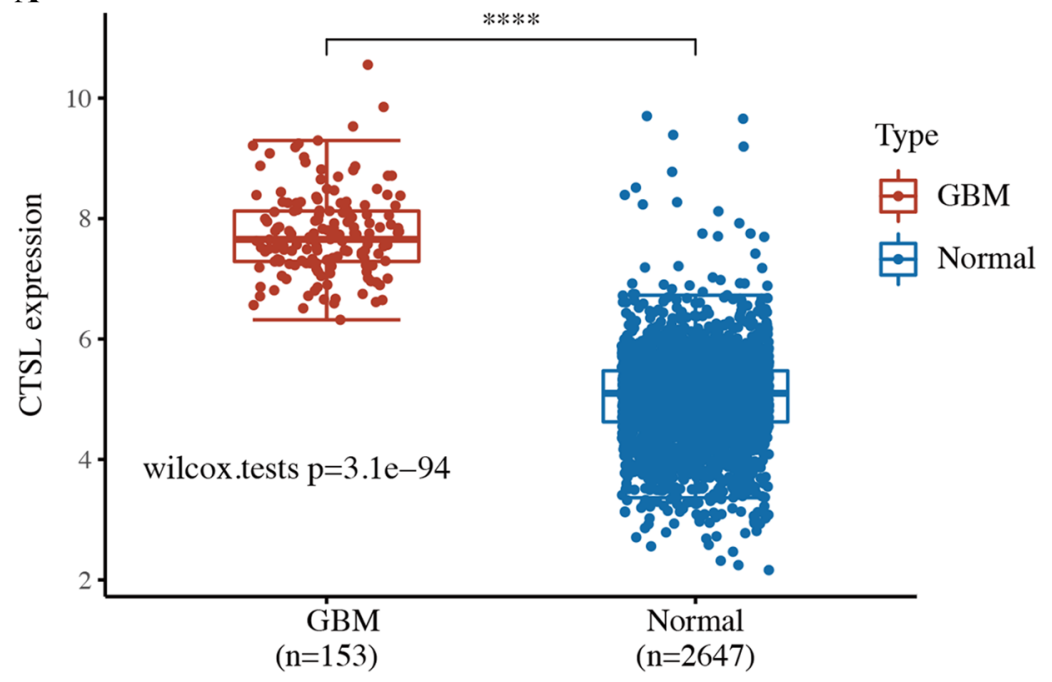

C

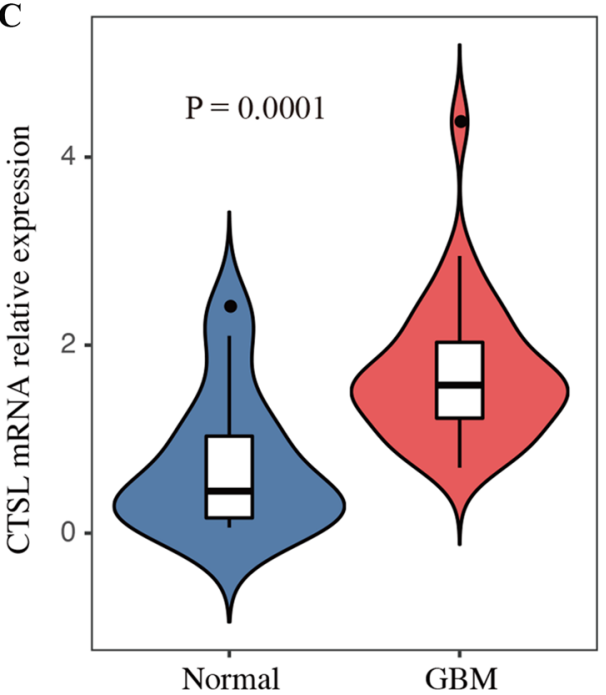

legend title

Normal

GBM
B

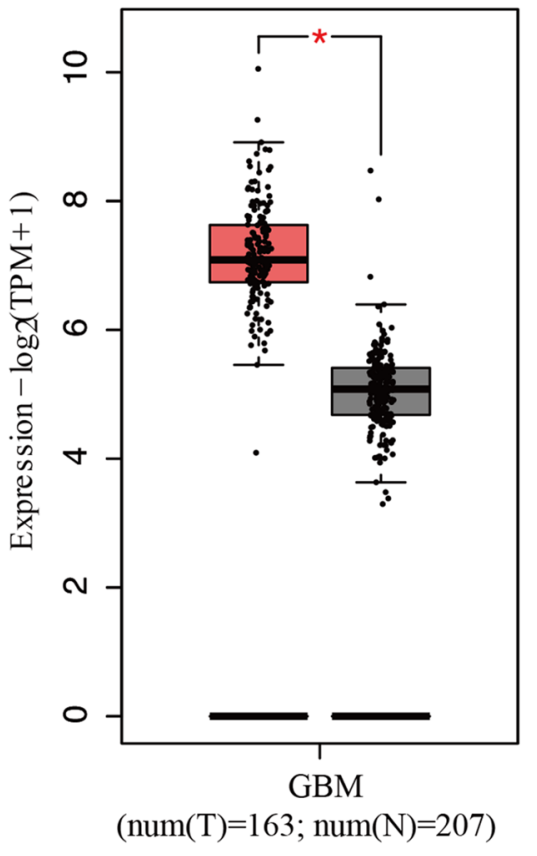

Fig. 1 The expression level of CTSL increases in GBM. A The CTSL mRNA expression level was analysis in TCGA database. B The CTSL mRNA expression level was analyzed in GEPIA database. C Expression of CTSL mRNA in GBM tissues were tested

Furthermore, the correlations between CTSL expression and major clinic pathological factors were determined using Cox regression analysis. Univariate Cox regression analysis revealed that CTSL expression was an independent prognostic factor for OS in patients with GBM (hazard ratio, 3.599; 95\% CI 1.260-10.278; $P=0.017$; Fig. 2F).

\section{Relationship between CTSL expression and tumor immune infiltration}

To have a better understanding of CTSL and the tumor immune microenvironment, we analysis the relationship between CTSL expression and tumor immune infiltration using TIMER database. Purity of the tumor means the tumor by RT-qPCR. D The protein expression levels of CTSL in patients with GBM were analyzed by western blotting. $* P<0.05$ and $* * * * P<0.001$

cell proportion in the tissue. The results showed that CTSL was very negatively correlated with purity $(r=-0.375$, $P<0.001)$, B cell $(r=-0.138, P<0.01)$ and $\mathrm{CD} 8^{+} \mathrm{T}$ cell $(r=-0.246, P<0.001)$. However, the expression of CTSL was positively correlated with the level of immune infiltration of dendritic cell neutrophil $(r=0.211, P<0.001)$ and dendritic cell $(r=0.63, P<0.001)$ in GBM (Fig 3$)$.

\section{Cathepsin inhibitor 1 reduced proliferation and invasion of U251 cells}

To evaluate the cytotoxic effect of cathepsin inhibitor 1 to GBM, U251 cells were seed in 96-well plates and treated with different concentration of BCA for 24,48 and $72 \mathrm{~h}$, and 
Table 1 Association of the expression level of CTSL mRNA with clinicopathological factors of glioma

\begin{tabular}{lllll}
\hline $\begin{array}{l}\text { Clinicopatho- } \\
\text { logical features }\end{array}$ & Patients $(n)$ & $\begin{array}{l}\text { CTSL mRNA expres- } \\
\text { sion }\end{array}$ & $P$ value \\
\cline { 3 - 4 } & & Low $(n)$ & High $(n)$ & \\
\hline Age & & & & \\
$>50$ & 20 & 11 & 9 & 0.826 \\
$\leq 50$ & 15 & 7 & 8 & \\
Gender & & & & \\
Male & 22 & 11 & 11 & 0.625 \\
Female & 13 & 7 & 6 & \\
KPS & & & & \\
$>80$ & 14 & 9 & 5 & 0.214 \\
$\leq 80$ & 21 & 9 & 12 & \\
\hline
\end{tabular}

KPS Karnofsky Performance Status

cell viability was measured using the CCK-8. As shown in Fig. 4A, cell viability decreased following treatment with cathepsin inhibitor 1 in a concentration-dependent manner. In addition, the Edu assay was performed to determine the effect of cathepsin inhibitor 1 on GBM cell proliferation. cathepsin inhibitor 1 treatment significantly increased the percentage of Edu-positive cells compared with the control (Fig. 4B). Taken together, these data indicated that cathepsin inhibitor 1 inhibited the growth of U251 cells in a concentration-dependent manner. To evaluate the effects of cathepsin inhibitor 1 on GBM cells migration, we performed wound healing assay. For this experiment, U251 cells were cultured and then co-incubated with different doses $(0,50$ and $100 \mu \mathrm{M})$ of CTSL- 1 for various time intervals $(0,12$ and $24 \mathrm{~h}$ ). Treatment with various doses of cathepsin inhibitor 1 for 12 and $24 \mathrm{~h}$ significantly decreased cell migration rates in U251 cells (Fig. 4C, D). We also examined cell migration and invasion capacity using a transwell chambers system after the indicated cell lines were treated with different doses $(0,50$ and $100 \mu \mathrm{M})$ of CTSL-1 (Fig. 4E, F, G). U251 cells significantly decreased migration and invasion rates significantly in a dose-dependent manner, which was consistent with the cell wound healing assay. These results showed that cathepsin inhibitor 1 inhibits U251 cell migration and invasion in vitro.

\section{Cathepsin inhibitor 1 induced apoptosis of U251 cells}

To verify whether CTSL-1 induced proliferation in an apoptosis-related manner, the effect of cathepsin inhibitor 1 on the apoptosis of U251 cells was examined using flow cytometry. We found that cathepsin inhibitor 1 treatment significantly increased the apoptosis of U251 cells (Fig. 5A, B). Meanwhile, we further detect molecular markers related to apoptosis. After treatment of cathepsin inhibitor 1, the expression of CTSL and anti-apoptosis protein BCL-2 expression markedly decrease, and pro-apoptosis protein Bax expression increase (Fig. 5C-G). Thus, these findings show that cytotoxic effects of cathepsin inhibitor 1 on U251 cells were partly caused by activation mitochondria-mediated intrinsic apoptotic pathway.

\section{Discussion}

In December 2019, a novel coronavirus (2019-nCov) has caused large-scale pandemics, which has caused a severe challenge for public health and the economy (Xie and Chen 2020). A large number of studies have described the mechanism by which the SARS-CoV-2 virus enters the cell. Although, it has been widely reported that SARSCoV2 promotes its entry into host cells through the spike protein that binds to the angiotensin-converting enzyme 2 (ACE-2) membrane-bound protein (Chen et al. 2020). CTSL seems to be responsible for the cleavage of S protein under different circumstances (Vargas-Alarcón et al. 2020). Therefore, understanding the regulation of viral entry in a comorbid state will also require understanding the expression of these genes. In this study, we analysis the CTSL mRNA level of GBM in GEPIA and TCGA database. We evaluated the relationship between the expression of CTSL and the prognosis of GBM patients who susceptibility to COVID-19. In addition, TIMER database was also used to analyze the correlation between CTSL and immune infiltration in GBM. Moreover, we used CTSL inhibitor to observe the effect on the proliferation, invasion, and apoptosis of GBM cells.

Recently studies demonstrated that cancer patients are confirmed to have a higher incidence of COVID-19, more severe symptoms and has a poor prognosis (Jacobo et al. 2020). SARS-CoV-2 can undergo endocytosis, endosomal maturation followed by cleavage with $\mathrm{pH}$-dependent cysteine protease CTSL to promote SARS-CoV-2 entry in cells. Recently study demonstrated that the down-regulation expression CTSL may be a potential mechanism that might reduce the ability of the virus to enter host cell, and regulation lysosome $\mathrm{PH}$ that would further interfere with proteolytic Spike protein activation may offer protection from the virus to enter (Kwan et al. 2021). So, CTSL plays a vital role in SARS-CoV-2 to enter cells. Here, our data demonstrated that CTSL upregulates in the GBM. In addition, SARS-CoV-2 might invade the 


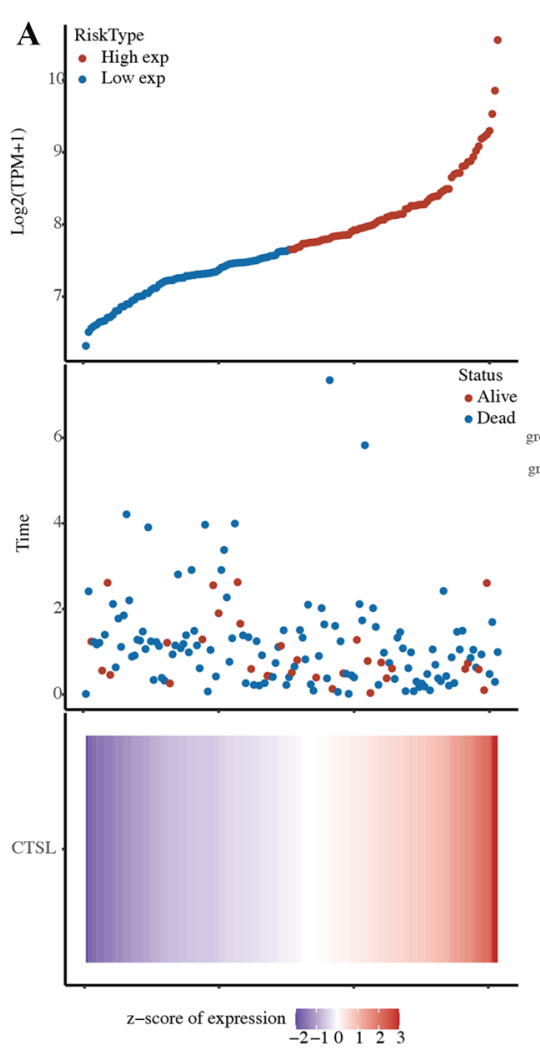

$\mathbf{F}$

\begin{tabular}{|c|c|c|}
\hline Characteristics & HR $(95 \% \mathrm{CI})$ & $P$ value \\
\hline Age $(<50$ vs $\geq 50)$ & $1.418(0.546-3.682)$ & 0.473 \\
\hline Gender (Male vs Female) & $1.637(0.602-4.454)$ & 0.334 \\
\hline $\operatorname{KPS}(<80$ vs $\geq 80)$ & $0.024(0.001-1.460) \oplus-4$ & 0.075 \\
\hline CTSL expression Low vs High & $3.599(1.260-10.278)$ & 0.017 \\
\hline
\end{tabular}

Fig. 2 Evaluation of the relationship between the CTSL and patient prognosis. A The curve of risk score. Survival status of the patients. More dead patients corresponding to the higher risk score. Heatmap of the expression profiles of CTSL in low- and high-expression group. B Kaplan-Meier survival analysis of CTSL in the TCGA set.

CNS, causing neurological disorders. There is currently no effective targeted drug to treat COVID-19. Although several countries have made great progress in researching COVID-19 vaccines, the vaccines are only effective for some person. A large number of studies have shown that some small molecule drugs have a good effect on the treatment of COVID-19, for example, melatonin (Zhou et al. 2020), hydroxychloroquine and chloroquine (Sanders et al. 2020). The main mechanism of these small molecule

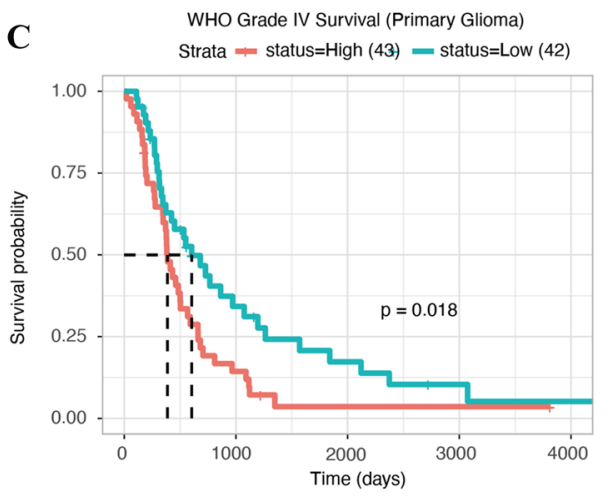

$\mathbf{E}$

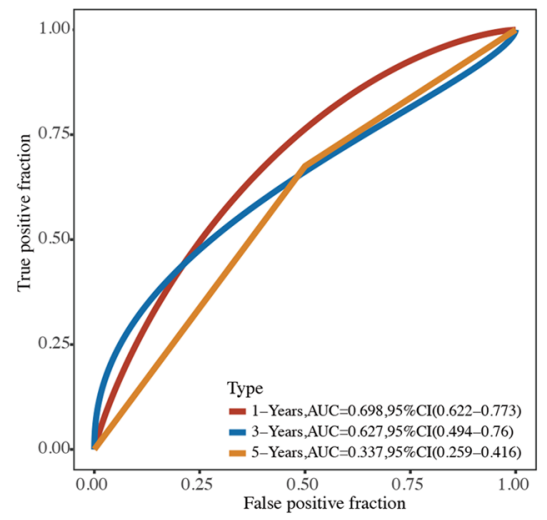

G

Multivariate cox analysis

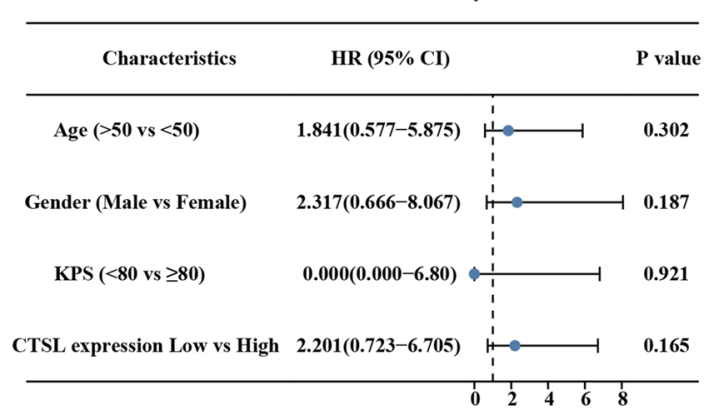

C Kaplan-Meier survival analysis of CTSL in the CGGA database. D Kaplan-Meier survival analysis of CTSL in the GBM tissue. E Timedependent ROC analysis of the CTSL in the TCGA set. F Univariate Cox regression forest map. $\mathbf{G}$ Multivariate Cox regression forest map

drugs is to inhibit virus entry by targeting the endocytic pathway (Merad and Martin 2020). Hydroxychloroquine and chloroquine increase the $\mathrm{pH}$ of the endosome, thereby inhibiting membrane fusion, which is a necessary mechanism for the virus to enter the cell (Savarino et al. 2006). In addition, the glycosylation of ACE2 and S protein can be inhibited to inhibit virus infection of cells.

Cancer is a disorder with immune dysfunction, and cancer patients are more susceptible to infections (Wang and Zhang 


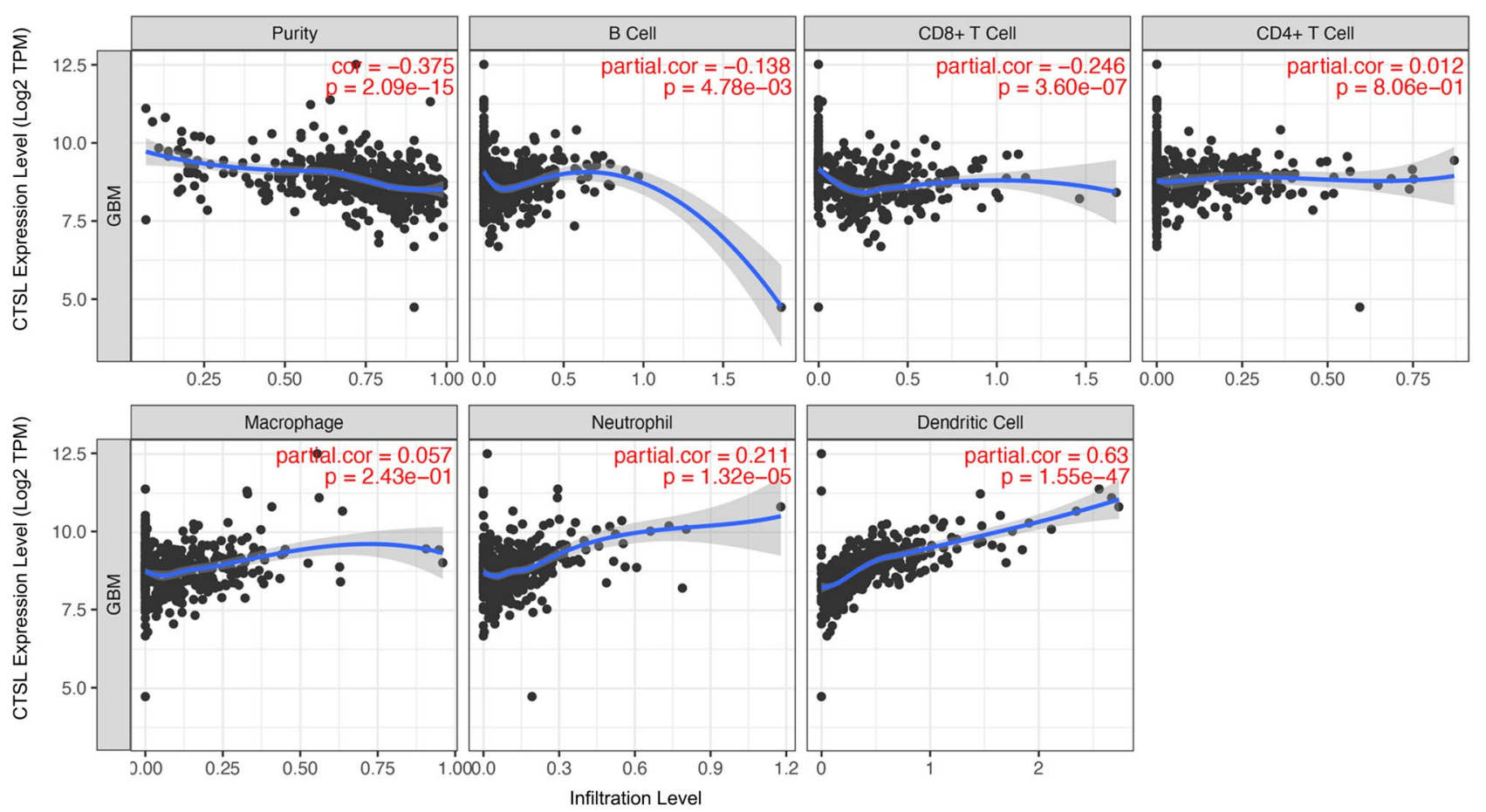

Fig. 3 Correlation between CTSL expression and tumor immune infiltration levels in GBM through TIMER database analysis

2020). Both humoral and cellular immunity are involved in the resistance to SARS-CoV-2 infection in the body. There is evidence that the abnormal regulation of immune response, especially $\mathrm{T}$ cells, may be highly related to the pathological process of COVID-19 (Qin et al. 2020). At the same time, others have also proved that abnormal and excessive immune cells (such as monocytes and macrophages) play a role in immune damage in COVID-19 (Merad and Martin 2020). We used the TIMER database to further explore its correlation with immune cell infiltration. The result showed that CTSL was very negatively correlated with purity, B cell and $\mathrm{CD}^{+} \mathrm{T}$ cell. However, the expression of CTSL was positively correlated with the level of immune infiltration of dendritic cell neutrophil and dendritic cell in GBM.

In addition, Kaplan-Meier curves and the log-rank test result showed that down regulation of CTSL had a favorable prognosis in GBM patients. Therefore, we evaluated the prognosis of GBM infected with SARS-CoV-2 according to the expression level of CTSL. Previous studies have found that CTSL highly expressed not only in gliomas but also in a variety of tumors, such as lung cancer, gastric cancer, ovarian cancer, breast cancer and other malignant tumors. So, cancer patients may be more susceptible to the SARS-CoV-2 infectious disease.

Previous studies have showed that CTSL expression is associated with the prognosis of tumor patients. For example, CTSL is over expression in nasopharyngeal carcinoma, breast cancer and pediatric acute myeloid leukemia, and is associated with poor disease-free survival (Yao et al. 2018; Sigloch et al. 2017). In this study, Kaplan-Meier survival analysis showed that patients with high CTSL levels had shorter OS and worse prognosis. Interestingly, evidence indicates that CTSL expression may be linked to the grade and stages of endometrial cancer patients. In addition, CTSL ablation can block angiogenesis in breast cancer by affecting cell cycle-associated genes, including cyclin D1-D3, E2, A2, B2 and H (Wang et al. 2019). Wang et al. (2016) demonstrated that Knockdown of Cathepsin L can inhibit GSC growth and increase tumor radio-sensitivity by decreasing CD133 expression and decrease DNA repair checkpoint proteins (ATM and DNA-PKcs). Qin et al. (2016) demonstrated that CTSL can promote proliferation of breast cancer and downregulation of CTSL significantly inhibited the proliferation of MCF-7 cells. Moreover, CTSL can inhibited A549 and MCF-7 cells migration by regulating PI3K-AKT and Wnt signaling pathways.

Importantly, evidence indicates that CTSL proteolytic activity is significantly higher in tumor tissue compared to normal tissue. In normal tissues, the extracellular matrix is protected by endogenous cathepsin inhibitors (such as cysteine and stefins) from undesirable proteolytic activity. 
A

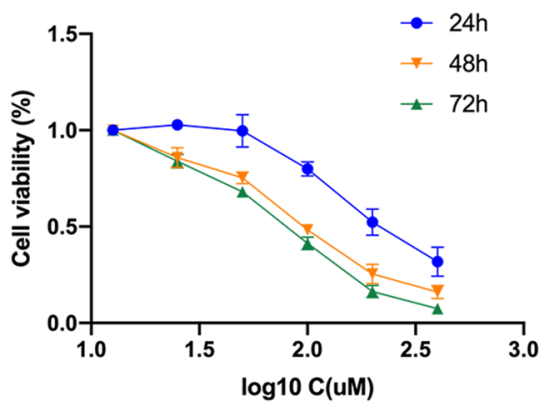

C

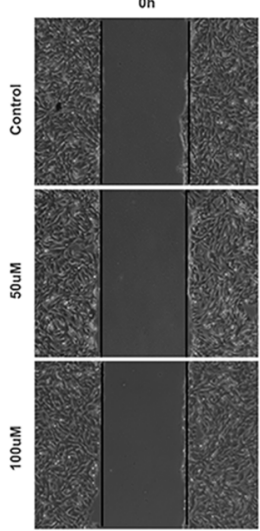

D

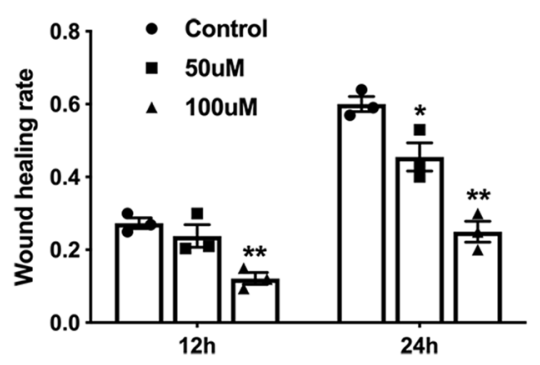

B

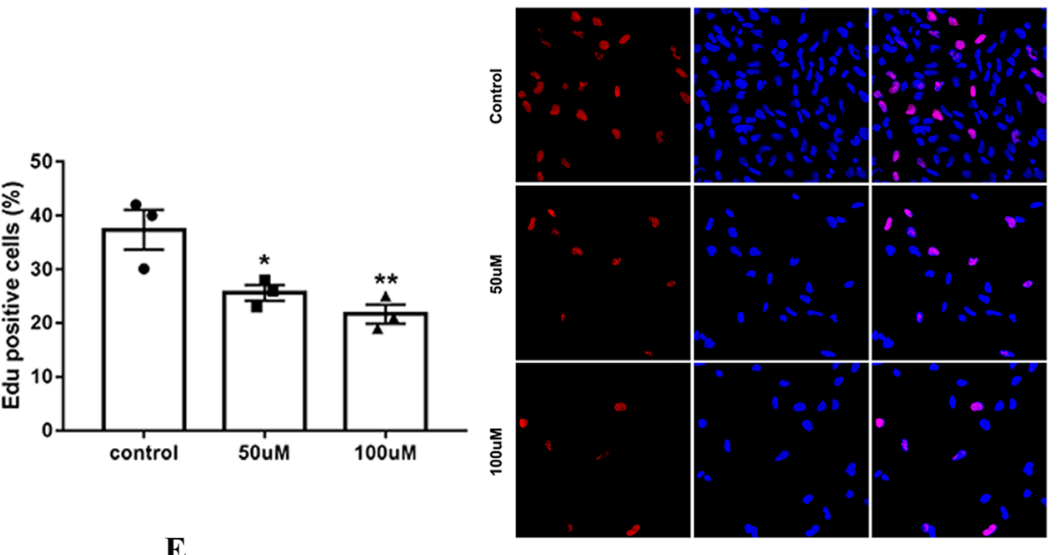

$24 \mathrm{~h}$

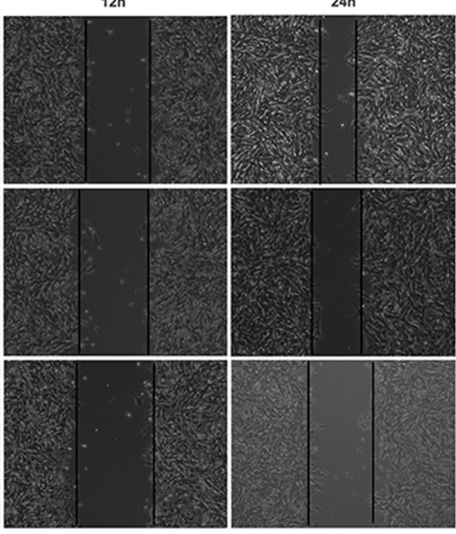

$\mathbf{F}$

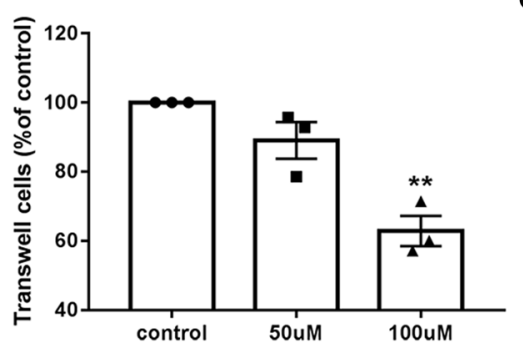

$\mathbf{E}$

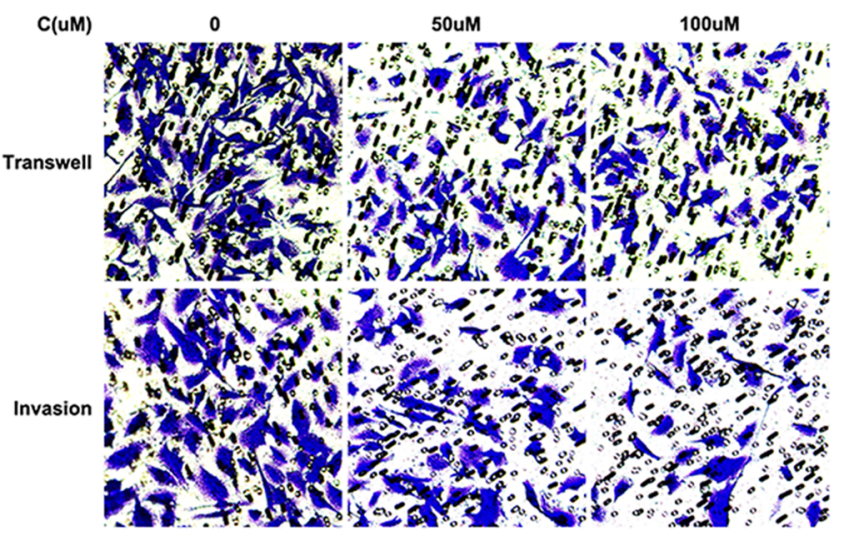

G

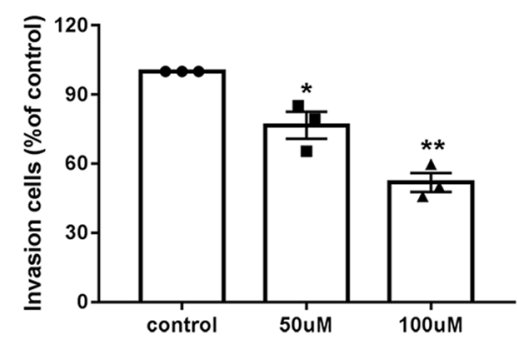

Fig. 4 Cathepsin inhibitor 1 inhibited the proliferation, migration, and invasion of U251 cells. A U251 cells were treated with various concentrations BCA for 24,48 and $72 \mathrm{~h}$. Cell proliferation was measured by CCK8 assay. B Cellular proliferation was measured via an Edu assay. $\mathbf{C}$ Wound healing assay shows the migrated cells at 0,12

However, studies have shown that the expression of these CTSL inhibitors declines sharply in various tumors and CTSL proteolytic activity increase. Therefore, the use of some exogenous CTSL inhibitors can reduce the expression and proteolytic activity of CTSL. For example, CTSL inhibitor KGP94 have anti-angiogenic efficacy, and can inhibited angiogenesis of breast cancer (Sudhan et al. 2016). Sudhan et al. (2016) found that KGP94 treatment significantly attenuation of tumor cell invasion and migration that it may have significant utility as an anti-metastatic agent. This study has found that CTSL inhibitor 1 significantly reduced the and $24 \mathrm{~h}$ after treatment with CTSL-1 (0, 50 and $100 \mu \mathrm{M})$. D Quantification of the wound healing rate in $\mathbf{A}$ after treatment with BCA. $\mathbf{E}$ After treatment, transwell assay showed that the migration and invasion cells at $24 \mathrm{~h}$. F, G Quantification of the migration and invasion cells. $* P<0.05$ and ${ }^{*} P<0.01$ for Student's $t$ test

growth, invasion, and migration of U251 cells in vitro and triggered mitochondrial apoptosis. Taken together, these findings suggest CTSL as a promising therapeutic target for clinical therapy in GBM patients. Therefore, because CTSL plays an important role in the pathogenesis of COVID-19, the targeted inhibition of CTSL may be an effective treatment option for this infectious disease.

In conclusion, higher expression of CTSL was found in GBM tissues compared with that of normal brain tissues, which resulted in significantly a poor survival rate in GBM 

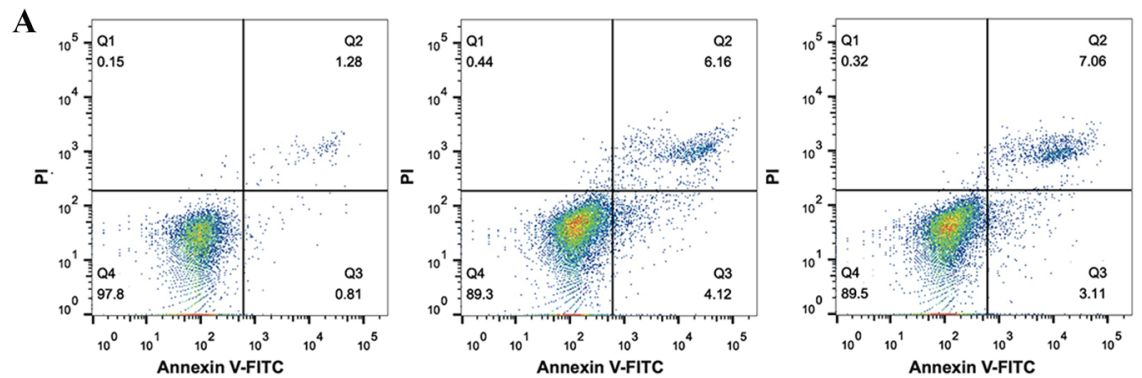

C

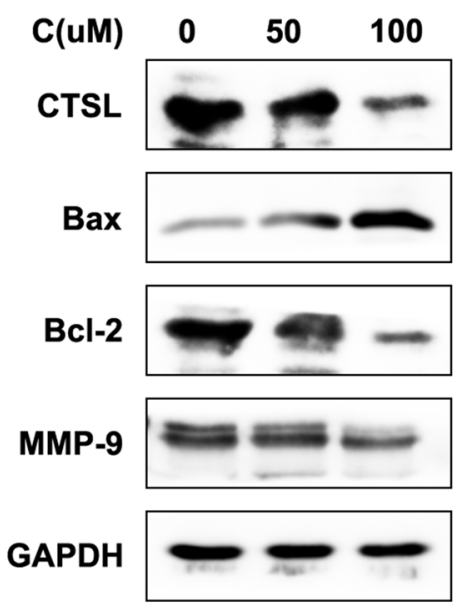

D

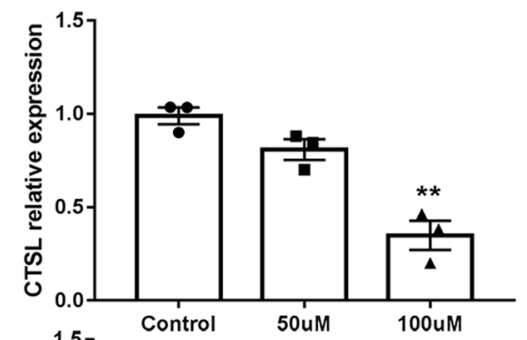

F

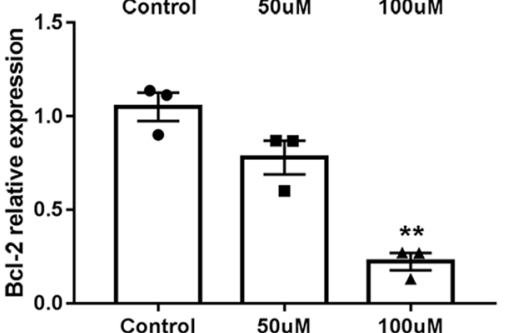

B

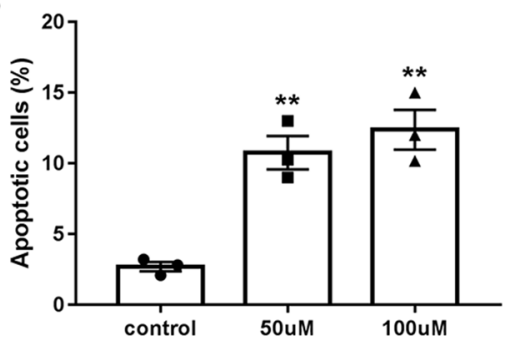

E

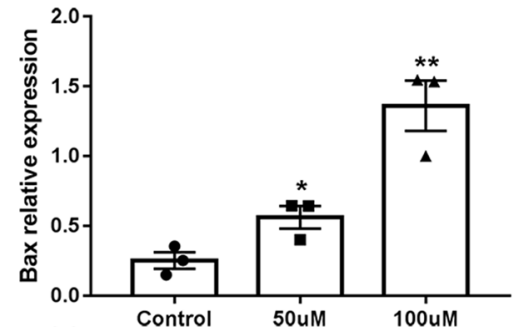

G

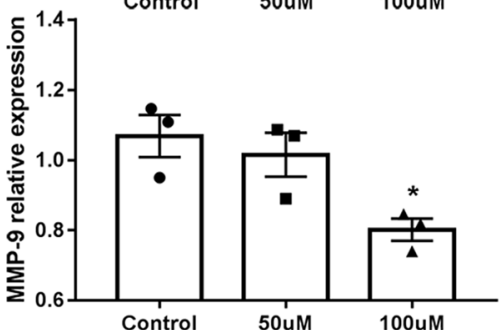

Fig. 5 Cathepsin inhibitor 1 promoted the apoptosis of U251 cells. A The apoptosis was measured by flow cytometry after treatment with 0,50 and $100 \mu \mathrm{M}$ cathepsin inhibitor 1 . B The percentage of cell apoptosis ratio in A. C Western blot analysis CTSL, MMP-9 Bax and
Bcl-2 expression in U251 cells treated with 0,50 and $100 \mu \mathrm{M}$ cathepsin inhibitor 1. D-G Analysis of relative expression levels of CTSL, MMP-9 Bax and Bcl-2 in $\mathbf{F} * P<0.05$ vs. Control group, $* * P<0.01$ vs. Control group patients. We considered the possible role of CTSL in the pathogenesis of COVID-19. Therefore, assessment of CTSL gene expression can be used to predict the prognosis and susceptibility to COVID-19 in these patient groups. CTSL inhibitors may be considered as promising treatments for GBM patients with SARS-CoV-2 infection.

Supplementary Information The online version contains supplementary material available at https://doi.org/10.1007/s00432-021-03843-9.

Acknowledgements This work was supported by grants from the National Natural Science Foundation of China (81960541/82060455), the Natural Science Foundation of Gansu Province (18JR3RA309 /18JR3RA365/20JR10RA741/20JR10RA766/21JR7RA426), the Science and Technology Research Project of Gansu Province (145RTSA012 and 17JR5RA307), the Project of Healty and Famliy Planing Commission of Gansu (GSWSKY-2014-31/ GSWSKY-2015-58/GSWSKY2018-01), the Lanzhou Science and Technology Bureau Project (2018-1-109), the Fundamental Research Funds for the Central University (lzujbky-2021-kb33), the Cuiying Science and Technology fund (CY2017-MS12/CY2017-MS15/CY2017BJ15/CYXZ-01), Cuiying Graduate Supervisor Applicant Training Program (201803) and Special fund project for doctoral training (YJSBD-13) of Lanzhou University Second Hospital.
Author contributions YP, GY and QD conceived the project. QD, QL, LD, HY, BW and LN performed the experiments. QD, QL, HW, YY, HZ and LN analyzed the data. QD, GY, QL, YY, BW and LD interpreted the data and revised the manuscript. YP, GY, QL, HW and QD wrote the manuscript. All authors read and approved the final manuscript.

Data availability The datasets generated for this study are available on request to the corresponding author.

\section{Declarations}

Conflict of interest The authors have no conflicts of interest to declare.

Ethics statement The animal study was reviewed and approved by Ethics Committee of the Lanzhou university Second hospital.

\section{References}

Achar A, Ghosh C (2020) COVID-19-associated neurological disorders: the potential route of cns invasion and blood-brain barrier relevance. Cells 9(11):2360 
Addeo A, Friedlaender A (2020) Cancer and COVID-19: unmasking their ties. Cancer Treat Rev 88:102041

Bao R, Hernandez K, Huang L, Luke JJ (2020) ACE2 and TMPRSS2 expression by clinical, HLA, immune, and microbial correlates across 34 human cancers and matched normal tissues: implications for SARS-COV-2 COVID-19. J Immunother Cancer 8(2): 001020

Brady CP, Brinkworth RI, Dalton JP, Dowd AJ, Verity CK, Brindley PJ (2000) Molecular modeling and substrate specificity of discrete cruzipain-like and cathepsin L-like cysteine proteinases of the human blood fluke Schistosoma mansoni. Arch Biochem Biophys 380(1):46-55

Chen K, Zou J, Han P, Han Z (2020) Single-cell RNA-seq data analysis on the receptor ACE2 expression reveals the potential risk of different human organs vulnerable to 2019-nCoV infection. Front Med 14(2): 185-192

Deng X, Lin D, Zhang X, Shen X, Yang Z, Yang L et al (2020) Profiles of immune-related genes and immune cell infiltration in the tumor microenvironment of diffuse lower-grade gliomas. J Cell Physiol 235(10):7321-7331

Gemmati D, Bramanti B, Serino ML, Secchiero P, Zauli G, Tisato V (2020) COVID-19 and individual genetic susceptibility/receptivity: role of ACE1/ACE2 genes, immunity, inflammation and coagulation. Might the double X-chromosome in females be protective against SARS-CoV-2 compared to the single X-chromosome in males? Int J Mol Sci 21(10):3474

Hoffmann M, Kleine-Weber H, Schroeder S, Krüger N, Phlmann S (2020) SARS-CoV-2 cell entry depends on ACE2 and TMPRSS 2 and is blocked by a clinically proven protease inhibitor. Cell 181(2):271-280

Hou Y, Zhao J, Martin W, Kallianpur A, Cheng F (2020) New insights into genetic susceptibility of COVID-19: an ACE2 and TMPRSS2 polymorphism analysis. BMC Med 18(1):216

Jacobo R, Pangua C, Serrano-Montero G, Obispo B, Lara MN (2020) COVID-19 and lung cancer: a greater fatality rate? Lung Cancer 146:19-22

Kwan J, Lin LT, Bell R, Bruce JP, Liu FF (2021) Elevation in viral entry genes and innate immunity compromise underlying increased infectivity and severity of COVID-19 in cancer patients. Sci Rep 11(1):4533

Leskowitz S, Phillipino L, Hendrick G, Graham JB (2015) Immune response in patients with cancer. Cancer 10(6):1103-1105

Merad M, Martin JC (2020) Pathological inflammation in patients with COVID-19: a key role for monocytes and macrophages. Nat Rev Immunol 20(6):1-8

Nakagawa T (1998) Cathepsin L: critical role in Ii degradation and CD4 T cell selection in the thymus. Science 280(5362):450-453

Narayanappa A, Chastain WH, Paz M, Solch RJ, Bix G (2021) SARSCoV-2 mediated neuroinflammation and the impact of COVID-19 in neurological disorders. Cytokine Growth Factor Rev 58(3):1-15

Qin G, Cai Y, Long J, Zeng H, Xu W, Li Y, Liu M, Zhang H, He ZL, Chen WG (2016) Cathepsin L is involved in proliferation and invasion of breast cancer cells. Neoplasma 63(1):30-36
Qin C, Zhou L, Hu Z, Zhang S, Tian DS (2020) Dysregulation of immune response in patients with COVID-19 in Wuhan, China. Clin Infect Dis 71(15):762-768

Sanders JM, Monogue ML, Jodlowski TZ, Cutrell JB (2020) Pharmacologic treatments for Coronavirus Disease 2019 (COVID-19): a review. J Am Med Assoc 323(18):1824-1836

Savarino A, Trani LD, Donatelli I, Cauda R, Cassone A (2006) New insights into the antiviral effects of chloroquine. Lancet Infect Dis 6(2):67-69

Shan Y, Yu Y, Wen Z, Wei Y, Liu T (2016) FOXO3a promotes gastric cancer cell migration and invasion through the induction of cathepsin L. Oncotarget 7(23):34773-34784

Sigloch FC, Tholen M, Gomez-Auli A, Biniossek ML, Reinheckel T, Schilling O (2017) Proteomic analysis of lung metastases in a murine breast cancer model reveals divergent influence of CTSB and CTSL overexpression. J Cancer 8(19):4065-4074

Sudhan DR, Siemann DW (2013) Cathepsin L inhibition by the small molecule KGP94 suppresses tumor microenvironment enhanced metastasis associated cell functions of prostate and breast cancer cells. Clin Exp Metastasis 30(7):891-902

Sudhan DR, Rabaglino MB, Wood CE, Siemann DW (2016) Cathepsin $\mathrm{L}$ in tumor angiogenesis and its therapeutic intervention by the small molecule inhibitor KGP94. Clin Exp Metastasis 33(5):461-473

Vargas-Alarcón G, Posadas-Sánchez R, Ramírez-Bello J (2020) Variability in genes related to SARS-CoV-2 entry into host cells (ACE2, TMPRSS2, TMPRSS11A, ELANE, and CTSL) and its potential use in association studies. Life Sci 260:118313

Wang H, Zhang L (2020) Risk of COVID-19 for patients with cancer. Lancet Oncol 21(4):e180

Wang W, Long LM, Wang L, Tan C, Feng X, Chen X, Liang Z (2016) Knockdown of Cathepsin L promotes radiosensitivity of glioma stem cells both in vivo and in vitro. Cancer Lett 371(2):274-284

Wang Z, Xiang Z, Zhu T, Chen J, Zhou W (2019) Cathepsin L interacts with CDK2AP1 as a potential predictor of prognosis in patients with breast cancer. Oncol Lett 19(1):167-176

Xie M, Chen Q (2020) Insight into 2019 novel coronavirus-an updated intrim review and lessons from SARS-CoV and MERSCoV. Int J Infect Dis 94:119-124

Yao F, Xiong Y, Xiao S, Zhao Y, Ying Z, Long W et al (2018) Cathepsin $\mathrm{L}$ promotes ionizing radiation-induced $\mathrm{U} 251$ glioma cell migration and invasion through regulating the GSK-3 $\beta / C U X 1$ pathway. Cell Signal 44:62

Zhou Y, Hou Y, Shen J, Huang Y, Cheng F (2020) Network-based drug repurposing for novel coronavirus 2019-nCoV/SARS-CoV-2. Cell Discov 16(6): 14

Publisher's Note Springer Nature remains neutral with regard to jurisdictional claims in published maps and institutional affiliations. 